\title{
Notching up hearing
}

Hair cells located within the organ of Corti in the cochlea of the inner ear are responsible for translating auditory stimuli into electromechanical signals that can be perceived by the nervous system. However, with ageing, acoustic trauma or following infection, these sensitive cells can become damaged, resulting in hearing loss. As hair cells do not regenerate, such deafness has been thought to be irreversible. Now, writing in Neuron, Mizutari et al. demonstrate that pharmacological inhibition of Notch signalling can partially restore hearing in mice exposed to acoustic trauma.

During embryonic development of the inner ear, prosensory epithelial cells acquire different fates - either hair cells or supporting cells through a process of lateral inhibition mediated by Notch signalling. Indeed, active Notch signalling on developing hair cells prevents adjacent supporting cells from differentiating into hair cells. With this in mind, the authors set out to determine whether new hair cells could be generated by manipulating Notch signalling.

Previously, the authors demonstrated that mouse inner ear stem cells isolated postnatally can act as hair cell precursors when Notch signalling was blocked using an inhibitor of gamma secretase - a proteolytic complex responsible for Notch activation. Therefore, they first screened several known gamma secretase inhibitors for their ability to induce hair cell differentiation from inner ear stem cells, and identified LY411575 as the most potent compound.

Next, they set out to characterize the effects of Notch inhibition in the organ of Corti. Treatment of mouse explant cultures of the neonatal organ of Corti with LY411575 induced extra hair cell differentiation. Moreover, in organ of Corti explants from mice in which outer hair cells had been ablated, LY411575 increased the number of hair cells in the outer hair cell region and decreased the number of supporting cells in the midapex and midbase of the cochlea, suggesting that supporting cells were transdifferentiating into hair cells.

Next, they exposed mice to an acoustic injury and assessed the effects of LY411575. Systemic oral administration of LY411575 caused significant side effects, therefore the compound was directly delivered into the inner ear. Notch signalling was transiently increased in the deafened mature cochlea following noise exposure, an effect that was blocked by LY411575. After 1 month of treatment, in vivo lineage tracing revealed that LY411575 promoted the transdifferentiation of supporting

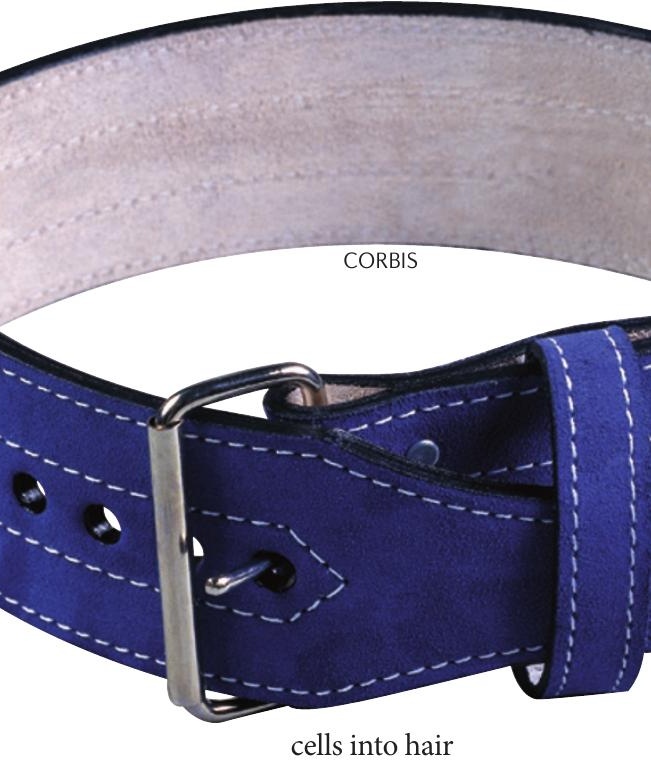
cells from the apical to midapical turn in the mature cochlea, and by 3 months the number of outer hair cells was increased throughout the middle of the cochlea. Importantly, measurement of the auditory brainstem response revealed partial recovery of hearing loss.

In summary, modulation of Notch signalling may represent a potential therapeutic approach for the treatment of deafness. Given that hair cell loss also underlies vestibular system disorders that affect the sense of balance, these findings may have implications for the treatment of several conditions.

Sarah Crunkhorn

ORIGINAL RESEARCH PAPER Mizutari, K. et al. Notch inhibition induces cochlear hair cell regeneration and recovery of hearing after acoustic trauma. Neuron 77, 58-69 (2013) 